\title{
Sugammadex: new questions on reversal
}

Recently released in Brazil, sugammadex is a modified $\gamma$-cyclodextrin, which is showing favorable results regarding reversal of motor blockade, particularly by rocuronium. One of the main advantages of this agent over neostigmine is the reversal of relaxation when the patient is deeply curarized. However, the use of sugammadex in such conditions of deep blockade has shown a phenomenon not previously seen: train-of-four (TOF) recovery before complete recovery of single twitch (T1), with a difference up to five minutes between the two types of neurostimulation ${ }^{1}$. The concept of a satisfactory recovery includes only the return of TOF $>0.9$ or, according to current guidelines, about $1.0^{2,3}$.

Neuromuscular transmission (NMT) monitoring is different from other methods used in anesthesia, such as pulse oximetry, because it requires the interpretation of data from the peripheral nerve stimulator.

For a better understanding, the NMT phenomena can be divided into three distinct parts: pre-synaptic processes; those related to the synaptic cleft and basement membrane; and post-synaptic or muscular. In the first, the greatest emphasis is on the alpha motor neurons in which neuronal nicotinic receptors can be identified ${ }^{4}$, as well as voltage-gated calcium and potassium channels, which are fundamental structures to control the entry of calcium into neuron. These receptors have characteristics that distinguish them from muscle receptors, such as the presence of only two types of subunits, $\alpha 2-10$ and $\beta 2-4$, and the lack of safety margin ${ }^{4-8}$. This last characteristic is related to the additional release of acetylcholine in the presence of high-intensity stimuli, by a positive feedback mechanism from stimulation of $\alpha 3 \beta 2$ nicotinic receptor.

When the neuronal receptor is occupied by a non-depolarizing neuromuscular blocker (NMB), the positive feedback mechanism and release of additional acetylcholine do not occur; and, in the presence of a high-intensity stimulus, the muscle does not maintain an intense contraction, i.e., shows fatigue. Other mechanisms, besides the blockade of neuronal nicotinic receptors, seem to be involved with the development of fatigue, as shown in muscle-phrenic nerve preparation. Among them, there are the facilitator action of type 1 muscarinic receptors (M1) and/or inhibitory action of type 2 (M2) ${ }^{9}$. In clinical monitoring this fatigue is characterized by TOF $<0.9^{2,10,11}$.

Physiologically, the acetylcholine molecules not destroyed in the synaptic cleft by acetylcholinesterase arrive at the muscular nicotinic receptor and occupy it triggering the opening of the receptor central pore, which is represented by M2-M4 chains located in the transmembrane portion of the sarcolemma ${ }^{4,8}$. Hydrated sodium molecules enter through this pore generating an action potential. The electric potential stimulates the sodium dependent voltage-gated receptors juxtaposed to muscular nicotinic receptors, which will allow the additional entry of sodium, increasing the action potential. This membrane depolarization releases intracellular calcium molecules, which trigger muscle contraction. Muscle contraction or the post-synaptic mechanism is assessed on the monitor by the response to the isolated stimulus, $T 1$.

In the presence of a non-depolarizing NMB that competes with acetylcholine for binding sites in muscular nicotinic receptors, there is reduction of muscle contraction represented by T1 depression, as the occupation surpasses the safety margin.

Succinylcholine, the only depolarizing NMB used in clinical practice, has no affinity for neuronal nicotinic receptors in conventional doses and, therefore, fatigue is not seen on monitor. Succinylcholine occupies post-synaptic receptors and decreases or abolishes muscle contraction, i.e., depresses or annul T1.

The administration of high doses of sugammadex to antagonize deep blockades promptly recovers TOF and, by definition, "releases" neuronal nicotinic receptors of the steroidal NMB, more specifically rocuronium. However, contrary to neostigmine, it does not promote reestablishment of $\mathrm{T} 1$ on the same proportion and speed, i.e., muscle receptors are still blocked by rocuronium. If we used the NMT monitor, this response follows the pattern of a partial succinylcholine block, i.e., depression of T1 and maintenance of TOF.

After these evidences, the following questions remain: Can "complete reversion" of NMB be considered with T1 depression still present? Why the rapid decrease of rocuronium molecule affects primarily neuronal receptors, exactly the ones with no safety margin? What are the effects of this drug that, on NMT monitor, shows the same pattern of a partial blockade with succinylcholine? What is the clinical significance of this reversal pattern?

NMT is a complex mechanism that remains unclear. Many explanations have been obtained from animal studies in which genetic manipulation resulting in modified animal allows the understanding of particular aspects of nerves and muscles and their occupation by venoms or xenobiotics. Perhaps, with this "new" reversal pattern after using Sugammadex, some concepts and mechanisms of NMB action on NMT, as well as those related to reversal, will be reviewed in Anesthesiology.

Maria Cristina S Almeida, PhD Universidade Federal de Santa Catarina

Giovani de Figueiredo Locks, MD, TSA Hospital Universitário

Universidade Federal de Santa Catarina 


\section{REFERENCES}

1. Staals LM, Driessen JJ, Van Egmond J, De Boer HD, Klimek M, Flockton EA, Snoeck MM - Train-of-four ratio recovery often precedes twitch recovery when neuromuscular block is reversed by sugammadex. Acta Anaesthesiol Scand, 2011;55:700-707.

2. Plaud B, Debaene B, Donati F, Marty J - Residual paralysis after emergence from anesthesia. Anesthesiology, 2010;112:1013-1022.
3. Capron F, Alla F, Hottier C, Meistelman C, Fuchs-Buder T - Can acceleromyography detect low levels of residual paralysis? A probability approach to detect a mechanomyographic train-of-four ratio of 0.9 . Anesthesiology, 2004;100:1119-1124.

4. Naguib M, Flood P, McArdle JJ, Brenner HR - Advances in neurobiology of the neuromuscular junction: implications for the anesthesiologist. Anesthesiology, 2002;96:202-231.

5. Faria M, Oliveira L, Timoteo MA, Lobo MG, Correia-De-Sa P - Blockade of neuronal facilitatory nicotinic receptors containing alpha 3 beta 2 subunits contribute to tetanic fade in the rat isolated diaphragm. Synapse, 2003;49:77-88.

6. Jonsson M, Gurley D, Dabrowski M, Larsson O, Johnson EC, Eriksson $\mathrm{LI}$ - Distinct pharmacologic properties of neuromuscular blocking agents on human neuronal nicotinic acetylcholine receptors: a possible explanation for the train-of-four fade. Anesthesiology, 2006;105:521533.

7. Changeux JP, Taly A - Nicotinic receptors, allosteric proteins and medicine. Trends Mol Med, 2008;14:93-102.

8. Unwin $\mathrm{N}$ - Refined structure of the nicotinic acetylcholine receptor at 4A resolution. J Mol Biol, 2005;346:967-989.

9. Bornia EC, Bando E, Machinski M, Jr., Pereira MW, Alves-Do-Prado $\mathrm{W}$ - Presynaptic M1, M2, and A1 receptors play roles in tetanic fade induced by pancuronium or cisatracurium. J Anesth, 2009;23:513519.

10. Kopman AF - Undetected residual neuromuscular block has consequences. Anesthesiology, 2008;109:363-364.

11. Kopman AF, Eikermann $M$ - Antagonism of non-depolarising neuromuscular block: current practice. Anaesthesia, 2009;64(Suppl1):2230 . 